\title{
Cat Gene
}

National Cancer Institute

\section{Source}

National Cancer Institute. cat Gene. NCI Thesaurus. Code C19450.

Bacterial chloramphenicol-O-acetyltransferase (cat) gene encodes chloramphenicol-Oacetyltransferase protein, an enzyme that acetylates the antiobiotic chloramphenicol to yield chloramphenicol 3-acetate. This product is not an inhibitor of peptidyltransferase, the enzyme responsible for natural chloramphenicol resistance in bacteria. The cat gene is used to select chloramphenicol-resistant colonies following genetic transfection of bacteria. 